\title{
Broken with Partner
}

National Cancer Institute

\section{Source}

National Cancer Institute. Broken with Partner. NCI Thesaurus. Code C150728.

The ending of a social partnership. 